\title{
Universal properties for universal types in bifibrational parametricity
}

\author{
Neil Ghani Fredrik Nordvall Forsberg Federico Orsanigo \\ University of Strathclyde, UK
}

Received December 2015, revised August 2017

In the 1980s, John Reynolds postulated that a parametrically polymorphic function is an ad-hoc polymorphic function satisfying a uniformity principle. This allowed him to prove that his set-theoretic semantics has a relational lifting which satisfies the Identity Extension Lemma and the Abstraction Theorem. However, his definition (and subsequent variants) have only been given for specific models. In contrast, we give a model-independent axiomatic treatment by characterising Reynolds' definition via a universal property, and show that the above results follow from this universal property in the axiomatic setting.

\section{Introduction}

A polymorphic function is parametric if its behavior is uniform across all of its type instantiations [Strachey, 2000]. Reynolds 1983] made this mathematically precise by formulating the notion of relational parametricity, and gave a set-theoretic model, where polymorphic programs are required to preserve all relations between instantiated types. Relational parametricity has proven to be one of the key techniques for formally establishing properties of software systems, such as representation independence Ahmed et al. 2009, Dreyer et al., 2012, equivalences between programs Hur and Dreyer, 2011, or deriving useful theorems about programs from their type alone Wadler, 1989.

In Reynolds' original model of parametricity, every type constructor $T$ of System F with $n$ free type variables is represented not just by a functor $\llbracket T \rrbracket_{0}: \mid$ Set $\left.\right|^{n} \rightarrow$ Set, but also by a functor $\llbracket T \rrbracket_{1}: \mid$ Rel $\left.\right|^{n} \rightarrow$ Rel, where the category Rel has as objects relations and as morphisms functions which preserve relatedness. Notice how both of these functors have as domain discrete categories; this ensures that (i) contravariant type expressions can be interpreted functorially; and (ii) that the functorial interpretation of function types can be defined pointwise. The interpretation is given by induction on the structure of the type $T$. When $T$ is a function type, say $T=U \rightarrow V$, we have

$$
\begin{aligned}
\llbracket U & \rightarrow V \rrbracket_{0} \vec{A}=\llbracket U \rrbracket_{0} \vec{A} \rightarrow \llbracket V \rrbracket_{0} \vec{A} \\
(f, g) \in \llbracket U & \rightarrow V \rrbracket_{1} \vec{R} \text { iff }(a, b) \in \llbracket U \rrbracket_{1} \vec{R} \Rightarrow(f a, g b) \in \llbracket V \rrbracket_{1} \vec{R}
\end{aligned}
$$

Not only are the above definitions empirically natural, but they are also supported by universal properties. Indeed, $\llbracket U \rightarrow V \rrbracket_{0}$ and $\llbracket U \rightarrow V \rrbracket_{1}$ are in fact exponential objects in 
their respective functor categories. The situation is less clear for $\forall$-types. If we denote the equality relation on the set $X$ by $\mathrm{Eq} X$, and lift that notation to tuples of types, then Reynolds interpretation of $\forall$-types is as follows:

$$
\begin{aligned}
& \llbracket \forall X . T \rrbracket_{0} \vec{A}=\left\{f: \prod_{X: \mathrm{Set}} \llbracket T \rrbracket_{0}(\vec{A}, X) \mid \forall R \in \operatorname{Rel}(A, B) .(f A, f B) \in \llbracket T \rrbracket_{1}(\mathrm{Eq} \vec{A}, R)\right\} \\
& (f, g) \in \llbracket \forall X . T \rrbracket_{1} \vec{R} \text { iff } \forall R \in \operatorname{Rel}(A, B) .(f A, g B) \in \llbracket T \rrbracket_{1}(\vec{R}, R)
\end{aligned}
$$

These definitions are empirically natural, conforming to the intuition that related inputs are mapped to related outputs. They work, in the sense that key theorems such as the Identity Extension Lemma and the Abstraction Theorem can be proved from them, but on the other hand, they lack a theoretical justification as to why they are the way they are. That is,

Are there universal properties underpinning the definition of $\llbracket \forall X . T \rrbracket_{0} \vec{A}$ and $\llbracket \forall X . T \rrbracket_{1} \vec{R}$ ? Can these universal properties be used to prove the Identity Extension Lemma and Abstraction Theorem in an axiomatic manner that is independent of specific models?

This paper answers the above questions positively for a large class of models axiomatically built from faithful bifibrations which admit full comprehension - this includes, for instance, subobject bifibrations. We believe this is of interest because the notion of a universal property is a fundamental mechanism used to give characterisations of key objects in mathematics, logic and computer science. Universal properties extract the core essence of structure.

Related work There is a significant body of work on the foundations of parametricity and, like us, many take a fibrational perspective. This can be traced back to the work of Hermida in his highly influential thesis 1993 and subsequent work Hermida, 2006. Other important work includes that of $\mathrm{Ma}$ and Reynolds [1992, who gave the first categorical framework for parametric polymorphism, Dunphy and Reddy 2004, who mixed fibrations with reflexive graphs, and Birkedal and Møgelberg [2005], who gave detailed and sophisticated models of not just parametricity, but of a first order logic on top of it in the form of Abadi-Plotkin Logic Plotkin and Abadi, 1993. Ghani et al. 2016b recently combined $\lambda 2$-fibrations and the fibrational notion of comprehension to give a sound and complete semantics of relational parametricity.

However, none of these papers tackles the question we tackle in this paper. Indeed, many follow the modern trend to bake in Identity Extension into their framework. In contrast, we dig deeper and prove the identity extension property from more primitive assumptions. Our own paper on parametric models Ghani et al. 2015a follows in the fibrational tradition, but distinguishes itself by using bifibrations. Since our work here requires bifibrations, this paper builds on the model presented there, and thus further validates this model.

Structure of the paper We first review Reynolds' model, and recast his definitions in a form suitable for generalisation in Section 2 We assume familiarity with category theory, but we give a brief introduction to fibrations in Section 3 , as well as our framework for 
models of System F. In Section 4 we first present the interpretation of $\forall$-types via a universal property. We prove that this definition satisfies the Identity Extension Lemma when instantiated to the subobject bifibration (Lemma 23), and the proof generalises to the case of faithful bifibrations which admit full comprehension (Lemma 28). The definition is further general enough to prove the Abstraction Theorem at the same time for both instantiations. Hence under appropriate assumptions, we have a parametric model of System F (Theorem 32), which implies that all the expected properties of parametricity hold. Finally we conclude in Section 5 with some comments on the hypothesis assumed in this work, and future work.

This paper is an extended version of a paper that appeared in the proceedings of WoLLIC 2015 Ghani et al. 2015b. This version includes the completely new Sections 3.3 and 4.3.2, which generalise the results from the conference version. In particular, while the conference version only proved that our universal property for parametrically polymorphic functions was sufficient to prove the Identity Extensional Lemma for subobject fibrations, this paper proves the result for a much broader class of fibrations - namely faithful bifibrations which admit full comprehension.

Acknowledgements We thank the anonymous referees for their constructive comments. This work was supported by EPSRC grants EP/K023837/1, Logical Relations for Program Verification, and EP/M016951/1, Homotopy Type Theory: Programming and Verification.

\section{Reynolds' Parametrically Polymorphic Functions}

We assume the reader is familiar with the syntax of System $\mathrm{F}$ and recall only those parts we need for our development — see e.g. Girard et al. 1989] for more details. In particular, the type judgements of System F are generated as follows:

$$
\frac{X_{i} \in \Gamma}{\Gamma \vdash X_{i} \text { Type }} \quad \frac{\Gamma \vdash T \text { Type } \Gamma \vdash V \text { Type }}{\Gamma \vdash T \rightarrow V \text { Type }} \quad \frac{\Gamma, X \vdash T \text { Type }}{\Gamma \vdash \forall X . T \text { Type }}
$$

where $\Gamma$ is a set of type variables. The term judgements of System $F$ are of the form $\Gamma ; \Delta \vdash t: T$ where $T$ is a System $\mathrm{F}$ type definable in the context $\Gamma$, and $\Delta$ is a term context associating distinct variables to a collection of types, each of which is also definable in $\Gamma$.

We write Set for the category of sets. Some care is needed here: in a metatheory using classical logic, there are no non-trivial set-theoretic parametric models of System F Reynolds, 1984. Instead, we should understand the category of sets e.g. internally to the Calculus of Constructions Coquand and Huet, 1988 with impredicative Set Atkey. 2009 (see also Pitts 1987 for other options). We further write Rel for the category whose objects are relations, i.e. subsets $R \subseteq A \times B$, and whose morphisms $(R \subseteq A \times B) \rightarrow$ $\left(R^{\prime} \subseteq A^{\prime} \times B^{\prime}\right)$ consist of functions $\left(f: A \rightarrow A^{\prime}, g: B \rightarrow B^{\prime}\right)$ such that if $(a, b) \in R$, then $(f a, g b) \in R^{\prime}$. In this case we say that the morphism in Rel is over the pair $(f, g)$. We write $U:$ Rel $\rightarrow$ Set $\times$ Set for the functor defined by $U(R \subseteq A \times B)=(A, B)$, which we note is faithful. If $F, G: \mid$ Set $\mid \rightarrow$ Set and $H: \mid$ Rel $\mid \rightarrow$ Rel are functors such that $U \circ H=(F \times G) \circ U$, then we say that $H$ is over $(F, G)$. If $\alpha: H \rightarrow H^{\prime}, \beta: F \rightarrow F^{\prime}$ and 
$\beta^{\prime}: G \rightarrow G^{\prime}$ are natural transformations between functors with $H$ over $(F, G)$ and $H^{\prime}$ over $\left(F^{\prime}, G^{\prime}\right)$, then we say that $\alpha$ is over $\left(\beta, \beta^{\prime}\right)$ if $U\left(\alpha_{R}\right)=\beta_{U R} \times \beta_{U R}^{\prime}$ for every $R$ in Rel.

Using formulas (1) and (2) from the introduction, Reynolds gives a two level semantics for System $\mathrm{F}$ where, if $\Gamma \vdash T$ Type and $|\Gamma|=n$, then $\llbracket T \rrbracket_{0}: \mid$ Set $\left.\right|^{n} \rightarrow$ Set and $\llbracket T \rrbracket_{1}:|\operatorname{Rel}|^{n} \rightarrow \operatorname{Rel}$ with $\llbracket T \rrbracket_{1}$ over $\left(\llbracket T \rrbracket_{0}, \llbracket T \rrbracket_{0}\right)$, i.e. if $\vec{R}: \operatorname{Rel}^{n}(\vec{A}, \vec{B})$, then $\llbracket T \rrbracket_{1} \vec{R}: \operatorname{Rel}\left(\llbracket T \rrbracket_{0} \vec{A}, \llbracket T \rrbracket_{0} \vec{B}\right)$. Reynolds also gives set-valued and relational interpretations of term contexts $\Delta=x_{1}: T_{1}, \ldots, x_{n}: T_{n}$ by defining $\llbracket \Delta \rrbracket_{0}=\llbracket T_{1} \rrbracket_{0} \times \cdots \times \llbracket T_{n} \rrbracket_{0}$ and $\llbracket \Delta \rrbracket_{1}=\llbracket T_{1} \rrbracket_{1} \times \cdots \times \llbracket T_{n} \rrbracket_{1}$ with $\llbracket \Delta \rrbracket_{1}$ over $\left(\llbracket \Delta \rrbracket_{0}, \llbracket \Delta \rrbracket_{0}\right)$. Reynolds then interprets each judgement $\Gamma ; \Delta \vdash t: T$ as a family of functions $\llbracket t \rrbracket_{0} S: \llbracket \Delta \rrbracket_{0} S \rightarrow \llbracket T \rrbracket_{0} S$ for each environment $S \in|\mathrm{Set}|^{|\Gamma|}$, and then proves the following theorems, which underpin most of the uses of parametricity.

Theorem 1 (Identity Extension Lemma). If $\Gamma \vdash T$ with $|\Gamma|=n$, then $\left(\llbracket T \rrbracket_{0}, \llbracket T \rrbracket_{1}\right)$ is equality-preserving, i.e. $\llbracket T \rrbracket_{1} \circ \mathrm{Eq}^{n}=\mathrm{Eq} \circ \llbracket T \rrbracket_{0}$.

Theorem 2 (Abstraction Theorem). If $\Gamma ; \Delta \vdash t: T$ with $|\Gamma|=n$, then for every $\vec{R}: \operatorname{Rel}^{n}(\vec{A}, \vec{B})$, if $(u, v) \in \llbracket \Delta \rrbracket_{1} \vec{R}$ then $\left(\llbracket t \rrbracket_{0} \vec{A} u, \llbracket t \rrbracket_{0} \vec{B} v\right) \in \llbracket T \rrbracket_{1} \vec{R}$.

So what makes Reynolds' definitions work? They are certainly fundamental, as can be seen by their numerous uses by programming language theorists (see e.g. Tse and Zdancewic [2004, Ahmed and Blume 2008, Ahmed et al. 2009, Hur and Dreyer [2011], Dreyer et al. 2012 ). While valuable, this only provides a partial answer, which ought to be complemented by a deeper and more fundamental understanding. For us, that takes the form of showing that the above definitions satisfy axiomatic universal properties, and that those universal properties are strong enough to prove key theorems such as Theorems 1 and 2 in that axiomatic setting. For function spaces, the answer is simply that $\llbracket T \rightarrow V \rrbracket_{0}$ is the exponential of the functors $\llbracket T \rrbracket_{0}$ and $\llbracket V \rrbracket_{0}$; and that $\llbracket T \rightarrow V \rrbracket_{1}$ is the exponential of the functors $\llbracket T \rrbracket_{1}$ and $\llbracket V \rrbracket_{1}$. These results in turn follow because Rel and Set are cartesian closed categories, and $U$ preserves this cartesian closed structure, i.e. products and exponentials of relations are again relations between products and exponentials, respectively. Our goal is to provide such a succinct and compelling equivalent explanation for the definitions of $\llbracket \forall X . T \rrbracket_{0} \vec{A}$ and $\llbracket \forall X . T \rrbracket_{1} \vec{R}$.

To begin with, note that if we were only to consider ad-hoc polymorphic functions, i.e. the collection

$$
\prod_{X: S e t} \llbracket T \rrbracket_{0}(\vec{A}, X)
$$

then we could characterise this collection as the product of the functor $\llbracket T \rrbracket_{0}(\vec{A},-)$ : Set $\rightarrow$ Set (naively assuming the product exists), that is, as the terminal $\llbracket T \rrbracket_{0}(\vec{A},-)$ cone. Including Reynolds' condition that a parametrically polymorphic function $f$ : $\prod_{S: \text { Set }} \llbracket T \prod_{0}(\vec{A}, S)$ is one where for every relation $R: \operatorname{Rel}(X, Y)$ we have that $(f X, f Y) \in$ $\llbracket T \rrbracket_{1}(\mathrm{Eq} \vec{A}, R)$ cuts down the number of ad-hoc polymorphic functions. Now the key bit. Define $\nu_{X}: \llbracket \forall X . T \rrbracket_{0} \vec{A} \rightarrow \llbracket T \rrbracket_{0}(\vec{A}, X)$ to be type application, i.e. $\nu_{X} f=f X$. Then Reynolds' parametricity condition that for all $R: \operatorname{Rel}(A, B)$, if $f: \llbracket \forall X . T \rrbracket_{0} \vec{A}$, then $(f A, f B) \in \llbracket T \rrbracket_{1}(\mathrm{Eq} \vec{A}, R)$ is equivalent to a morphism Eq $\left(\llbracket \forall X . T \rrbracket_{0} \vec{A}\right) \rightarrow \llbracket T \rrbracket_{1}(\mathrm{Eq} \vec{A}, R)$ over $\nu_{A}$ and $\nu_{B}$. Generalising, we have: 
Definition 3. Let $F=\left(F_{0}, F_{1}\right)$ be a pair of functors with $F_{0}$ : $\mid$ Set $\mid \rightarrow$ Set and $F_{1}$ : $|\operatorname{Re}| \rightarrow$ Rel such that $F_{1}$ is over $\left(F_{0}, F_{0}\right)$. An $F$-eqcone is an $F_{0}$-cone $\left(A: \operatorname{Set}, \nu: A \rightarrow F_{0}\right)$ such that there exists a (necessarily unique since $U$ is faithful) $F_{1}$-cone (Eq $A, \eta: \mathrm{Eq} A \rightarrow F_{1}$ ) with vertex $\mathrm{Eq} A$, and $\eta$ over $(\nu, \nu)$. The category of such cones is the full subcategory of $F_{0}$-cones whose objects are $F$-eqcones.

Our axiomatic definition is linked to Reynolds' definition in the following way:

Theorem 4. Assume $\Gamma, X \vdash T$ Type. For every tuple $\vec{A}$, Reynolds' set of parametrically polymorphic functions $\llbracket \forall X . T \rrbracket_{0} \vec{A}$ from $(2 \rrbracket$ is the terminal $F$-eqcone for the pair of functors $F=\left(\llbracket T \rrbracket_{0}(\vec{A},-), \llbracket T \rrbracket_{1}(\mathrm{Eq} \vec{A},-)\right)$.

Proof. Application at $X$, defined by $\nu_{X} f=f X$, makes $\llbracket \forall X . T \rrbracket_{0} \vec{A}$ a vertex of a $\llbracket T \rrbracket_{0}(\vec{A},-)$-cone. The uniformity condition on elements of $\llbracket \forall X . T \rrbracket_{0} \vec{A}$ ensures this cone is an $F$-eqcone. To see that this is the terminal such, consider any other $F$-eqcone $(A, \eta)$. As this is a $\llbracket T \rrbracket_{0}(\vec{A},-)$-cone, there is a unique map $\bar{\eta}$ of such cones into $\prod_{X: S e t} \llbracket T \rrbracket_{0}(\vec{A}, X)$. However, the fact that $(A, \eta)$ is an $F$-eqcone means the image of this mediating map lies within $\llbracket \forall X . T \rrbracket_{0} \vec{A}$. Hence we have a morphism of $F$-eqcones $A \rightarrow \llbracket \forall X . T \rrbracket_{0} \vec{A}$. The uniqueness of this mediating morphism follows from the uniqueness of $\bar{\eta}$.

We can also give a universal property to characterise $\llbracket \forall X . T \rrbracket_{1} \vec{R}$.

Definition 5. Let $F=\left(F_{0}, F_{1}\right)$ and $G=\left(G_{0}, G_{1}\right)$ be pairs of functors $\mid$ Set $\mid \rightarrow$ Set and $\mid$ Rel $\mid \rightarrow$ Rel with $F_{1}$ over $\left(F_{0}, F_{0}\right), G_{1}$ over $\left(G_{0}, G_{0}\right)$, and let $H: \mid$ Rel $\mid \rightarrow$ Rel with $H$ over $\left(F_{0}, G_{0}\right)$. A fibred $(F, G, H)$-eqcone consists of an $F$-eqcone $\left(A, \nu: A \rightarrow F_{0}\right)$, a $G$-eqcone $\left(B, \mu: B \rightarrow G_{0}\right)$ and a $H$-cone $(Q, \gamma: Q \rightarrow H)$ over $(\nu, \mu)$. The category of such cones has as morphisms triples $(f, g, h)$, where $f$ is a morphism between the underlying $F$-eqcones, $g$ is a morphism between the underlying $G$-eqcones and $h$ is a (again necessarily unique) morphism of $H$-cones above $(f, g)$.

The above definition can be understood as follows. For every relation $R: \operatorname{Rel}(X, Y)$ we need two things to be related, which is forced by $\gamma$. That the related things are instances of polymorphic functions is reflected by the fact that $\gamma_{R}$ is over $\left(\nu_{X}, \mu_{Y}\right)$. This intuition can be formalised via the following theorem:

Theorem 6. Assume $\Gamma, X \vdash T$ Type. For every relation $\vec{R}: \operatorname{Rel}(\vec{A}, \vec{B})$, the relation $\llbracket \forall X . T \rrbracket_{1} \vec{R}$ from $(2)$ is the terminal fibred $(F, G, H)$-eqcone for the functors $F=$ $\left(\llbracket T \rrbracket_{0}(\vec{A},-), \llbracket T \rrbracket_{1}(\mathrm{Eq} \vec{A},-)\right), G=\left(\llbracket T \rrbracket_{0}(\vec{B},-), \llbracket T \rrbracket_{1}(\mathrm{Eq} \vec{B},-)\right)$ and $H=\llbracket T \rrbracket_{1}(\vec{R},-)$.

Proof. Straightforward calculation, similar to the proof of Theorem 4

The Identity Extension Lemma and the Abstraction Theorem follow from these universal properties, as we will see in Theorems 23 and 32 in Section 4 . First, we turn to our axiomatic setting for the study of parametricity. 


\section{Fibrational Tools}

The previous section only covered a specific model, but what we really want is an axiomatic approach which can then be instantiated. There are a number of axiomatic approaches to parametricity, e.g. Ma and Reynolds [1992, Dunphy and Reddy 2004, Birkedal and Møgelberg 2005], Hermida [2006], Ghani et al. 2016b]. As we shall see, our axiomatisation requires a bifibration, and for this reason, we build upon our own treatment Ghani et al. 2015a, whose distinguishing feature is exactly bifibrational structure.

\subsection{Fibrations: an introduction}

We give a brief introduction to fibrations; for more details see Jacobs 1999 .

Definition 7. Let $U: \mathcal{E} \rightarrow \mathcal{B}$ be a functor. A morphism $g: Q \rightarrow P$ in $\mathcal{E}$ is cartesian over $f: X \rightarrow Y$ in $\mathcal{B}$ if $U g=f$ and, for every $g^{\prime}: Q^{\prime} \rightarrow P$ in $\mathcal{E}$ with $U g^{\prime}=f \circ v$ for some $v: U Q^{\prime} \rightarrow X$, there exists a unique $h: Q^{\prime} \rightarrow Q$ with $U h=v$ and $g^{\prime}=g \circ h$. Dually, a morphism $g: P \rightarrow Q$ in $\mathcal{E}$ is opcartesian over $f: X \rightarrow Y$ in $\mathcal{B}$ if $U g=f$ and, for every $g^{\prime}: P \rightarrow Q^{\prime}$ in $\mathcal{E}$ with $U g^{\prime}=v \circ f$ for some $v: Y \rightarrow U Q^{\prime}$, there exists a unique $h: Q \rightarrow Q^{\prime}$ with $U h=v$ and $g^{\prime}=h \circ g$.

Definition 8. A functor $U: \mathcal{E} \rightarrow \mathcal{B}$ is a fibration if for every object $P$ of $\mathcal{E}$ and every morphism $f: X \rightarrow U P$ in $\mathcal{B}$, there is a cartesian morphism $g: Q \rightarrow P$ in $\mathcal{E}$ over f. Similarly, $U$ is an opfibration if for every object $P$ of $\mathcal{E}$ and every morphism $f: U P \rightarrow Y$ in $\mathcal{B}$, there is an opcartesian morphism $h: P \rightarrow Q$ in $\mathcal{E}$ over $f$. A functor $U$ is a bifibration if it is both a fibration and an opfibration.

Let $U: \mathcal{E} \rightarrow \mathcal{B}$ be a fibration. We say that $U$ is cloven if it comes with a choice of cartesian liftings, i.e., if for every morphism $f: X \rightarrow Y$ in $\mathcal{B}$ and every object $P$ in $\mathcal{E}$ with $U(P)=Y$, there is a chosen cartesian morphism $f_{P}^{\S}: f^{*} P \rightarrow P$. Dually, an opfibration $U: \mathcal{E} \rightarrow \mathcal{B}$ is cloven if for every morphism $f: X \rightarrow Y$ in $\mathcal{B}$ and every object $P$ in $\mathcal{E}$ with $U(P)=X$, there is a chosen opcartesian morphism $f_{\S}^{P}: P \rightarrow \Sigma_{f} P$. A bifibration is cloven if it is cloven both as a fibration and as an opfibration. If $U$ is a cloven fibration or opfibration, then we write $f_{P}^{\S}$ for the cartesian morphism over $f$ with codomain $P$, and $f_{\S}^{P}$ for the opcartesian morphism over $f$ with domain $P$ respectively. We write $f^{*} P$ for the domain of $f_{P}^{\S}$ and $\Sigma_{f} P$ for the codomain of $f_{\S}^{P}$.

In this paper, we will generally assume that fibrations and opfibrations are cloven.

Example 9. Consider a category $\mathcal{B}$ with pullbacks, and let $\operatorname{Sub}_{\mathcal{B}}(A)$ be the category of subobjects of $A \in \mathcal{B}$ (i.e. equivalence classes of monos $m: X \hookrightarrow A)$. Let $\operatorname{Sub}(\mathcal{B})$ be the category with objects pairs $(A, m)$ where $m$ is in $\operatorname{Sub}_{\mathcal{B}}(A)$. A morphism $(f, \alpha)$ : $(A, m: X \hookrightarrow A) \rightarrow(B, n: Y \hookrightarrow B)$ consists of morphisms $f: A \rightarrow B$ and $\alpha: X \rightarrow Y$ in $\mathcal{B}$ such that $f \circ m=n \circ \alpha$. The functor $U: \operatorname{Sub}(\mathcal{B}) \rightarrow \mathcal{B}$ defined by $U(A, m)=A$ is then a fibration (with reindexing given by pullback), and further a bifibration if $\mathcal{B}$ has image factorisations. This fibration is cloven if $\mathcal{B}$ has chosen pullbacks. For $\mathcal{B}=$ Set, subobjects of $A$ can be identified with subsets of $A$.

If $U: \mathcal{E} \rightarrow \mathcal{B}$ is a fibration, opfibration, or bifibration, then $\mathcal{E}$ is its total category and $\mathcal{B}$ 
is its base category. An object $P$ in $\mathcal{E}$ is over its image $U P$ and similarly for morphisms. A morphism is vertical if it is over id. We write $\mathcal{E}_{X}$ for the fibre over an object $X$ in $\mathcal{B}$, i.e., the subcategory of $\mathcal{E}$ of objects over $X$ and vertical morphisms. For each morphism $f: X \rightarrow Y$ in $\mathcal{B}$ in a cloven fibration, the function mapping an object $P$ of $\mathcal{E}$ to $f^{*} P$ extends to a reindexing functor $f^{*}: \mathcal{E}_{Y} \rightarrow \mathcal{E}_{X}$. Dually, in a cloven opfibration, the function mapping an object $P$ of $\mathcal{E}_{X}$ to $\Sigma_{f} P$ extends to an opreindexing functor $\Sigma_{f}: \mathcal{E}_{X} \rightarrow \mathcal{E}_{Y}$. The assignments $f \mapsto f^{*}$ and $f \mapsto \Sigma_{f}$ are functorial up to isomorphism, i.e. id $\cong$ id, $f^{*} \circ g^{*} \cong(g \circ f)^{*}, \Sigma_{\text {id }} \cong$ id and $\Sigma_{g} \circ \Sigma_{f} \cong \Sigma_{g \circ f}$. When these isomorphisms are identities, we say that we have a split fibration, opfibration, or bifibration respectively.

We write $|\mathcal{E}|$ for the discrete subcategory of $\mathcal{E}$. If $U: \mathcal{E} \rightarrow \mathcal{B}$ is a functor, then the discrete functor $|U|:|\mathcal{E}| \rightarrow|\mathcal{B}|$ is induced by the restriction of $U$ to $|\mathcal{E}|$, and is always a bifibration. If $n \in \mathbb{N}$, then $\mathcal{E}^{n}$ denotes the $n$-fold product of $\mathcal{E}$ in Cat. The $n$-fold product of $U$, denoted $U^{n}: \mathcal{E}^{n} \rightarrow \mathcal{B}^{n}$, is the functor defined by $U^{n}\left(X_{1}, \ldots, X_{n}\right)=\left(U X_{1}, \ldots, U X_{n}\right)$. If $U$ is a fibration or opfibration, then so is $U^{n}$.

\subsection{Fibrations of relations}

Since Reynolds' theory of parametricity is about relations, we describe relations in a fibrational setting. If $U$ is a fibration whose base has products, then the associated fibration of relations $\operatorname{Rel}(U)$ is obtained by change of base along the product functor, i.e. the following pullback:

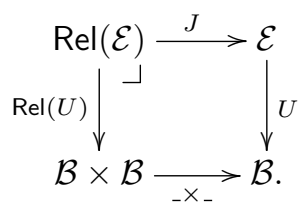

If $U$ is a fibration or opfibration, then so is $\operatorname{Rel}(U)$. The bifibration $\operatorname{Rel} \rightarrow$ Set $\times$ Set from Section 2 arises as the fibration of relations associated to the subobject fibration $\operatorname{Sub}($ Set $) \rightarrow$ Set from Example 9 . The map $U \mapsto \operatorname{Rel}(U)$ can be extended, for every category $\mathcal{B}$ which has products, to a functor Rel : $\operatorname{Fib}_{\mathcal{B}} \rightarrow \mathrm{Fib}_{\mathcal{B} \times \mathcal{B}}$ from the category of fibrations over $\mathcal{B}$ and fibred functors to the category of fibrations over $\mathcal{B} \times \mathcal{B}$ and fibred functors, but we will not make essential use of this fact in the current paper.

To treat equality in this axiomatic framework, we first need the notion of truth. Let $U: \mathcal{E} \rightarrow \mathcal{B}$ be a fibration with (chosen) fibred terminal objects, i.e. each fibre $\mathcal{E}_{X}$ has a (chosen) terminal object $K X$, and reindexing preserves it. Then the assignment $X \mapsto K X$ extends to the functor $K: \mathcal{B} \rightarrow \mathcal{E}$, called the truth functor. This functor is right adjoint to $U$. Furthermore the counit from $U \circ K$ to Id is the identity, and $K$ is full and faithful. Equality arises axiomatically as follows:

Lemma 10 (Lawvere 1970]). Let $U: \mathcal{E} \rightarrow \mathcal{B}$ be a bifibration with fibred terminal objects. Assume $\mathcal{B}$ has products, and write $\delta_{A}=\left\langle\mathrm{id}_{A}, \mathrm{id}_{A}\right\rangle: A \rightarrow A \times A$. The map $A \mapsto \Sigma_{\delta_{A}} K A$ extends to a functor $\mathrm{Eq}: \mathcal{B} \rightarrow \operatorname{Rel}(\mathcal{E})$, called the equality functor.

Example 11. The subobject fibration from Example 9 has fibred terminal objects given 
by $K A=\mathrm{id}_{A}: A \hookrightarrow A$. Opreindexing by a mono is by composition in the subobject fibration, hence equality is given by $\mathrm{Eq} A=\delta_{A}: A \hookrightarrow A \times A$.

\subsection{Fibrations which admit full comprehension}

We have seen in the previous section how relations can be treated abstractly as objects $(A, B, R)$ in the total category of a certain fibration. Of course, a more concrete - but less general - approach to relations is to consider them to be spans in the base category of the fibration.

Definition 12. Let $\mathcal{B}$ be a category. The category $\operatorname{Span}(\mathcal{B})$ of spans in $\mathcal{B}$ has as objects pairs of morphisms with the same domain

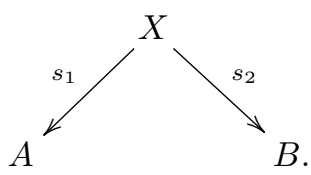

A morphism consists of a triple $(f, g, h):\left(A \stackrel{s_{1}}{\longleftarrow} X \stackrel{s_{2}}{\longrightarrow} B\right) \rightarrow\left(A^{\prime} \stackrel{s_{1}^{\prime}}{\longleftarrow} X^{\prime} \stackrel{s_{2}^{\prime}}{\longrightarrow} B^{\prime}\right)$ where $f: X \rightarrow X^{\prime}, g: A \rightarrow A^{\prime}$ and $h: B \rightarrow B^{\prime}$ are morphisms in $\mathcal{B}$ such that $s_{1}^{\prime} \circ f=g \circ s_{1}$ and $s_{2}^{\prime} \circ f=h \circ s_{2}$. Alternatively $\operatorname{Span}(\mathcal{B})$ is the functor category $B^{\cdot \leftarrow \cdot \rightarrow}$.

A natural question is whether the abstract, fibrational notion of relation behaves sufficiently like the concrete, span-based relations so that theorems concerning the latter can be generalised to the former. The answer is that, yes, this is possible if more structure is present. This structure is known as comprehension and is used widely in categorical logic Jacobs, 1999]. We will give an example of a generalisation of a result using comprehension in Section 4.3.2. In the current section, we recall the definitions and basic properties, and explore a formal relationship between fibrational relations and spans: comprehension guarantees the existence of the functor $\operatorname{Rel}(\mathcal{P})$ below - if furthermore the comprehension is full, then $\operatorname{Rel}(\mathcal{P})$ has a left adjoint $\mathcal{L}$ :

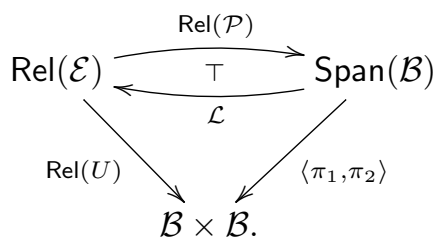

At a formal level, this adjunction between $\operatorname{Rel}(\mathcal{E})$ and $\operatorname{Span}(\mathcal{B})$ allows the transfer of results mentioned above, but we will not pursue this further in later sections in this article. We note in passing that much of this structure arises from applying Rel $: \mathrm{Fib}_{\mathcal{B}} \rightarrow \mathrm{Fib}_{\mathcal{B} \times \mathcal{B}}$ 
to the diagram

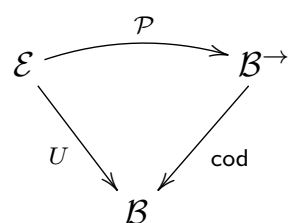

up to the isomorphism $\operatorname{Rel}\left(\mathcal{B}^{\rightarrow}\right) \cong \operatorname{Span}(\mathcal{B})$. Here, comprehension again guarantees the existence of $\mathcal{P}$, and full comprehension implies that $\mathcal{P}$ has a left adjoint $\mathcal{B} \rightarrow \rightarrow \mathcal{E}$.

Definition 13 (Ehrhard [1988]). Let $U: \mathcal{E} \rightarrow \mathcal{B}$ be a fibration. The fibration $U$ admits comprehension if it has a truth functor $K$, and $K$ further has a right adjoint $\{-\}: \mathcal{E} \rightarrow \mathcal{B}$, called the comprehension functor.

Note that Ehrhard 1988 uses the terminology D-category for the data making up a fibration admitting comprehension, while Jacobs 1993 uses comprehension category with unit. The following well-known result shows how comprehension allows objects in the total category of a fibration to be seen as morphisms in the base category - this foreshadows our own result showing how objects in the total category of a fibration of relations can be seen as spans in the base category.

Lemma 14 (Jacobs 1993). Let $U: \mathcal{E} \rightarrow \mathcal{B}$ be a fibration admitting comprehension. Comprehension $\{-\}$ induces a functor $\mathcal{P}: \mathcal{E} \rightarrow \mathcal{B}^{\rightarrow}$ defined by $\mathcal{P}(X)=U\left(\epsilon_{X}\right)$, where $\epsilon$ is the unit of the adjunction $K \dashv\{-\}$. For $f: X \rightarrow Y$ in $\mathcal{E}, \mathcal{P}(f)$ is defined by $\mathcal{P}(f)=(\{f\}, U(f))$. Furthermore, the assignment $\pi_{X}:=U\left(\epsilon_{X}\right)$ is a natural transformation $\pi:\{-\} \rightarrow U$.

Proof. Note that the construction of $\pi$ relies on $U \circ K=$ Id. Naturality of $\pi$ follows by applying $U$ to the naturality of $\epsilon$. The functor $\mathcal{P}$ arises as any natural transformation induces a functor into the arrow category.

We can adapt the above lemma to the setting of relations and spans as follows:

Lemma 15. Let $U: \mathcal{E} \rightarrow \mathcal{B}$ be a fibration admitting comprehension. Comprehension $\{-\}$ induces a functor $\operatorname{Rel}(\mathcal{P}): \operatorname{Rel}(\mathcal{E}) \rightarrow \operatorname{Span}(\mathcal{B})$.

Proof. An object $(A, B, R)$ of $\operatorname{Rel}(\mathcal{E})$ is by definition an object $R$ of $\mathcal{E}_{A \times B}$. The action of $\mathcal{P}$ gives a morphism $\pi_{R}:\{R\} \rightarrow A \times B$ which is an object of $\operatorname{Span}(\mathcal{B})$. The action of $\operatorname{Rel}(\mathcal{P})$ on morphisms is defined similarly.

The following stronger property will be used in Section 4.3 .2

Definition 16 (Jacobs 1993). A fibration $U: \mathcal{E} \rightarrow \mathcal{B}$ admits full comprehension if $U$ admits comprehension, and the functor $\mathcal{P}: \mathcal{E} \rightarrow \mathcal{B}^{\rightarrow}$ induced by $\left\{{ }_{-}\right\}$from Lemma 14 is full and faithful.

In the remainder of this section, we show that full comprehension for a faithful fibration implies that $\operatorname{Rel}(\mathcal{P}): \operatorname{Rel}(\mathcal{E}) \rightarrow \operatorname{Span}(\mathcal{B})$ has a left adjoint. The candidate functor $\mathcal{L}:$ $\operatorname{Span}(\mathcal{B}) \rightarrow \operatorname{Rel}(\mathcal{E})$ can be defined using weaker assumptions: 
Lemma 17. Let $U: \mathcal{E} \rightarrow \mathcal{B}$ be a bifibration with truth functor $K$. The map which sends $A \stackrel{s_{1}}{\longleftarrow} X \stackrel{s_{2}}{\longrightarrow} B$ in $\operatorname{Span}(\mathcal{B})$ to $\left(A, B, \Sigma_{\left\langle s_{1}, s_{2}\right\rangle} K X\right)$ extends to a functor $\mathcal{L}: \operatorname{Span}(\mathcal{B}) \rightarrow$ $\operatorname{Rel}(\mathcal{E})$.

Proof. Given a morphism $(f, g, h):\left(A \stackrel{s_{1}}{\longleftarrow} X \stackrel{s_{2}}{\longrightarrow} B\right) \rightarrow\left(A^{\prime} \stackrel{s_{1}^{\prime}}{\longleftarrow} X^{\prime} \stackrel{s_{2}^{\prime}}{\longrightarrow} B^{\prime}\right)$ in Span(B), $\mathcal{L}(f, g, h)$ is defined using the universal property of $\left\langle s_{1}, s_{2}\right\rangle_{K X}^{\S}$ in the following diagram

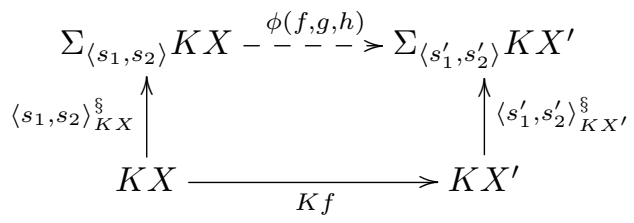

over the diagram

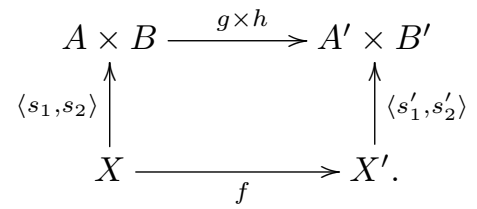

in $\mathcal{B}$, which commutes since $(f, g, h)$ is a morphism in $\operatorname{Span}(\mathcal{B})$.

Working towards the adjunction $\mathcal{L} \dashv \operatorname{Rel}(\mathcal{P})$, we first need a simple technical result.

Lemma 18. Let $U: \mathcal{E} \rightarrow \mathcal{B}$ be a fibration admitting comprehension. For every $A$ in $\mathcal{B}$, the morphism $\pi_{K A}$ is an isomorphism $\pi_{K A}:\{K A\} \cong A$.

Proof. Since $K$ is full and faithful, the unit $\eta$ of the adjunction $K \dashv\{-\}$ is a natural isomorphism. Using the triangle identity $\epsilon_{K A} \circ K\left(\eta_{A}\right)=$ id we have $\epsilon_{K A}=K\left(\eta_{A}\right)^{-1}$. Finally $\pi_{K A}=U\left(\epsilon_{K A}\right):\{K A\} \cong A$.

We can now show that $\mathcal{L}$ actually is left adjoint to $\operatorname{Rel}(\mathcal{P})$, assuming a little extra structure:

Theorem 19. Let $U: \mathcal{E} \rightarrow \mathcal{B}$ be a faithful bifibration with truth functor $K$ and full comprehension. Then $\mathcal{L} \dashv \operatorname{Rel}(\mathcal{P})$.

Proof. We describe a natural isomorphism

$$
\operatorname{Rel}(\mathcal{E})\left(\mathcal{L}\left(A \stackrel{s_{1}}{\longleftarrow} X \stackrel{s_{2}}{\longrightarrow} B\right),\left(A^{\prime}, B^{\prime}, R^{\prime}\right)\right) \cong \operatorname{Span}(\mathcal{B})\left(\left(A \stackrel{s_{1}}{\longleftarrow} X \stackrel{s_{2}}{\longrightarrow} B\right), \operatorname{Rel}(\mathcal{P})\left(A^{\prime}, B^{\prime}, R^{\prime}\right)\right) .
$$

Given $(f, g, \alpha):\left(A, B, \Sigma_{\left\langle s_{1}, s_{2}\right\rangle} K X\right) \rightarrow\left(A^{\prime}, B^{\prime}, R^{\prime}\right)$ in $\operatorname{Rel}(\mathcal{E})$, consider the composition $\alpha \circ\left\langle s_{1}, s_{2}\right\rangle^{\S}: K X \rightarrow R^{\prime}$ in $\mathcal{E}$. By applying $\mathcal{P}$, we obtain the commuting diagram

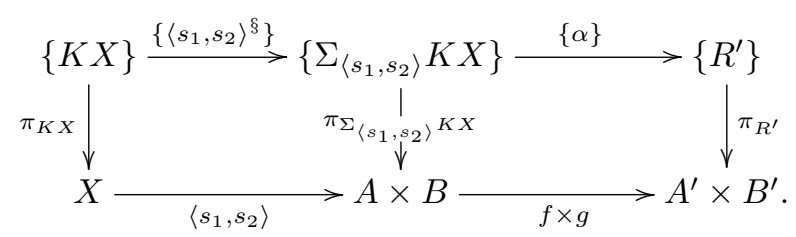


and since $\pi_{K X}$ is an isomorphism by Lemma 18 , this gives a morphism from $\left\langle s_{1}, s_{2}\right\rangle: X \rightarrow$ $A \times B$ to $\pi_{R^{\prime}}:\left\{R^{\prime}\right\} \rightarrow A^{\prime} \times B^{\prime}$. We hence map $(f, g, \alpha)$ to $\left(\left\{\alpha \circ\left\langle s_{1}, s_{2}\right\rangle^{\S}\right\} \circ \pi_{K X}^{-1}, f, g\right)$.

In the other direction, consider $\left(f, f_{0} \times f_{1}\right):\left\langle s_{1}, s_{2}\right\rangle \rightarrow \pi_{R^{\prime}}$ in $\operatorname{Span}(\mathcal{B})$. Using the universal property of $\left\langle s_{1}, s_{2}\right\rangle^{\S}$ we obtain

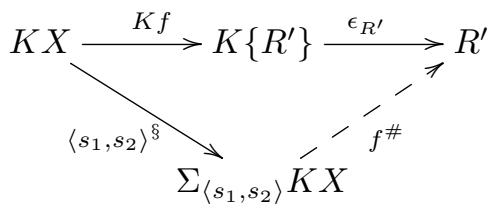

over the diagram

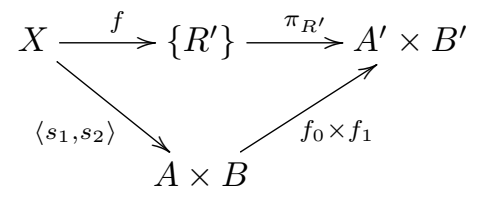

in $\mathcal{B}$, which commutes since $\left(f, f_{0}, f_{1}\right)$ is a morphism in $\operatorname{Span}(\mathcal{B})$. We map $\left(f, f_{0} \times f_{1}\right)$ to $\left(f_{0}, f_{1}, f^{\#}\right)$.

It remains to check that these maps are each other's inverses. In one direction, this amounts to checking that $\alpha=\left(\left\{\alpha \circ\left\langle s_{1}, s_{2}\right\rangle^{\S}\right\} \circ \pi_{K X}^{-1}\right)^{\#}$, which follows since $\alpha$ satisfies the universal property defining $\left(\left\{\alpha \circ\left\langle s_{1}, s_{2}\right\rangle^{\S}\right\} \circ \pi_{K X}^{-1}\right)$, by naturality of $\epsilon_{R}$. In the other direction, we need to show that $f=\left\{f^{\#} \circ\left\langle s_{1}, s_{2}\right\rangle^{\S}\right\} \circ \pi_{K X}^{-1}$. These morphisms respectively make the following diagrams commute:
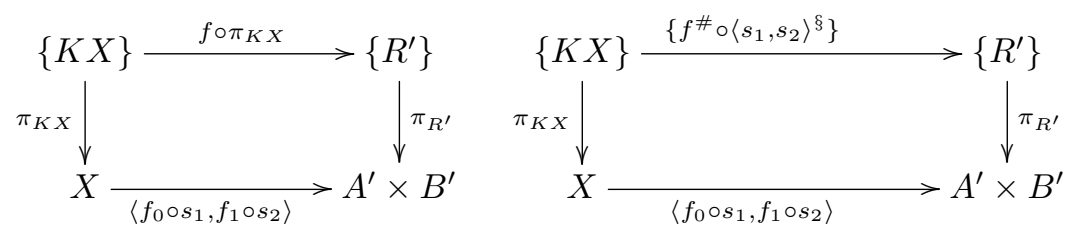

By fullness of $\mathcal{P}$, there exists $\rho, \rho^{\prime}: K X \rightarrow R^{\prime}$ with $\{\rho\}=f \circ \pi_{K X}, U(\rho)=\left\langle f_{0} \circ s_{1}, f_{1} \circ s_{2}\right\rangle$, $\left\{\rho^{\prime}\right\}=\left\{f^{\#} \circ\left\langle s_{1}, s_{2}\right\rangle^{\S}\right\}$ and $U\left(\rho^{\prime}\right)=\left\langle f_{0} \circ s_{1}, f_{1} \circ s_{2}\right\rangle$. By faithfulness of $U, \rho=\rho^{\prime}$ and hence $f=\{\rho\} \circ \pi_{K X}^{-1}=\left\{\rho^{\prime}\right\} \circ \pi_{K X}^{-1}=\left\{f^{\#} \circ\left\langle s_{1}, s_{2}\right\rangle^{\S}\right\} \circ \pi_{K X}^{-1}$ as required.

\subsection{Parametricity: a model using bifibrations}

Let $U: \mathcal{E} \rightarrow \mathcal{B}$ and $U^{\prime}: \mathcal{E}^{\prime} \rightarrow \mathcal{B}^{\prime}$ be fibrations. A fibred functor $T: U \rightarrow U^{\prime}$ comprises two functors $T_{0}: \mathcal{B} \rightarrow \mathcal{B}^{\prime}$ and $T_{1}: \mathcal{E} \rightarrow \mathcal{E}^{\prime}$ such that $T_{1}$ is over $T_{0}$, i.e. $U^{\prime} \circ T_{1}=T_{0} \circ U$, and $T_{1}$ preserves cartesian morphisms. If $T^{\prime}: U \rightarrow U^{\prime}$ is another fibred functor, then a fibred natural transformation $\nu: T \rightarrow T^{\prime}$ comprises two natural transformations $\nu_{0}: T_{0} \rightarrow T_{0}^{\prime}$ and $\nu_{1}: T_{1} \rightarrow T_{1}^{\prime}$ such that $U^{\prime} \nu_{1}=\nu_{0} U$. Note that in the case of fibred functors $|\operatorname{Rel}(U)| \rightarrow \operatorname{Rel}(U)$, the requirement that cartesian morphisms are preserved is vacuous. Nevertheless, we will avoid introducing more terminology and stick with 'fibred' also in this case.

Armed with these definitions, we can introduce the axiomatic framework for parametricity within which we can generalise the universal properties of Section 2 . Given a 
bifibration $U: \mathcal{E} \rightarrow \mathcal{B}$ with appropriate structure (see Section 4.4), we interpret types $\Gamma \vdash T$ as fibred functors $\llbracket T \rrbracket=\left(\llbracket T \rrbracket_{0} \times \llbracket T \rrbracket_{0}, \llbracket T \rrbracket_{1}\right):|\operatorname{Rel}(\mathcal{E})|^{|\Gamma|} \rightarrow \operatorname{Rel}(\mathcal{E})$, and terms $\Gamma ; \Delta \vdash t: T$ as fibred natural transformations $\left(\llbracket t \rrbracket_{0} \times \llbracket t \rrbracket_{0}, \llbracket t \rrbracket_{1}\right): \llbracket \Delta \rrbracket \rightarrow \llbracket T \rrbracket$. Thus a type $T$ has a "standard" semantics $\llbracket T \rrbracket_{0}$, as well as a relational semantics $\llbracket T \rrbracket_{1}$. The interpretation can be summed up as follows:

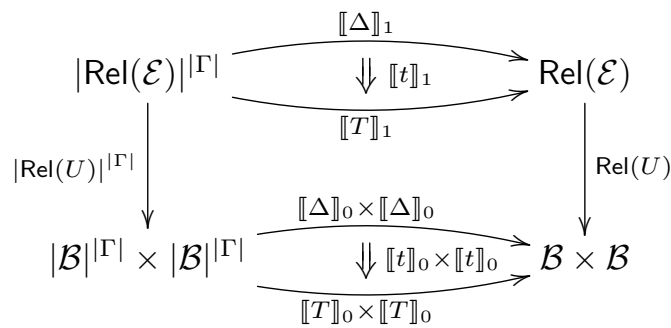

In Reynolds' model, the Abstraction Theorem states that if $\vec{R}:|\operatorname{Re}(\mathcal{E})| \Gamma \mid(\vec{A}, \vec{B})$, and $(u, v) \in \llbracket \Delta \rrbracket_{1} \vec{R}$, then $\left(\llbracket t \rrbracket_{0} \vec{A} u, \llbracket t \rrbracket_{0} \vec{B} v\right) \in \llbracket T \rrbracket_{1} \vec{R}$. This is equivalent to a natural transformation $\llbracket t \rrbracket_{1}$ over $\left(\llbracket t \rrbracket_{0}, \llbracket t \rrbracket_{0}\right)$. Thus the existence of $\llbracket t \rrbracket_{1}$ is the fibrational analogue of the Abstraction Theorem. See Ghani et al. 2015a for more details.

\section{Parametrically Polymorphic Functions, Axiomatically}

We now turn to the universal property we will use to define the object of parametrically polymorphic functions in our axiomatic framework. We carefully formulated the definitions of Section 2 so that they seamlessly generalise once we have axiomatic notions of relations and equality, which we developed in Section 3 . We assume for the rest of the paper that the fibration $U: \mathcal{E} \rightarrow \mathcal{B}$ is faithful. We discuss this assumption in the conclusion in Section 5

\subsection{Eqcones and fibred eqcones}

We start by generalising the definition of eqcones for a faithful fibration $U: \mathcal{E} \rightarrow \mathcal{B}$ and the fibration of relations $\operatorname{Rel}(U): \operatorname{Rel}(\mathcal{E}) \rightarrow \mathcal{B} \times \mathcal{B}$ obtained via change of base from $U$.

Definition 20. Let $F=\left(F_{0}, F_{1}\right)$ be a pair of functors with $F_{0}:|\mathcal{B}| \rightarrow \mathcal{B}$ and $F_{1}:|\operatorname{Rel}(\mathcal{E})| \rightarrow \operatorname{Rel}(\mathcal{E})$ such that $F_{1}$ is over $\left(F_{0}, F_{0}\right)$. An $F$-eqcone is an $F_{0}$-cone $(A, \nu$ : $\left.A \rightarrow F_{0}\right)$ such that there exists a (necessarily unique since $U$ is faithful) $F_{1}$-cone (Eq $A, \eta$ ) with vertex $\mathrm{Eq} A$, and $\eta$ over $(\nu, \nu)$. The category of such cones is the full subcategory of $F_{0}$-cones whose objects are $F$-eqcones. We denote the terminal object of this category $\forall_{0} F$, if it exists.

The universal property defining the relational interpretation of parametrically polymorphic functions smoothly generalises also to the fibrational setting:

Definition 21. Let $F=\left(F_{0}, F_{1}\right)$ and $G=\left(G_{0}, G_{1}\right)$ be pairs of functors $|\mathcal{B}| \rightarrow \mathcal{B}$ and $|\operatorname{Rel}(\mathcal{E})| \rightarrow \operatorname{Rel}(\mathcal{E})$ with $F_{1}$ over $\left(F_{0}, F_{0}\right), G_{1}$ over $\left(G_{0}, G_{0}\right)$, and let $H:|\operatorname{Rel}(\mathcal{E})| \rightarrow \operatorname{Rel}(\mathcal{E})$ with $H$ over $\left(F_{0}, G_{0}\right)$. A fibred $(F, G, H)$-eqcone consists of an $F$-eqcone $\left(A, \nu: A \rightarrow F_{0}\right)$, 
a $G$-eqcone $\left(B, \mu: B \rightarrow G_{0}\right)$ and an $H$-cone $(Q, \gamma: Q \rightarrow H)$ such that $Q$ is over $A \times B$, and $\gamma$ is over $(\nu, \mu)$. A morphism $(A, \nu, B, \mu, Q, \gamma) \rightarrow\left(A^{\prime}, \nu^{\prime}, B^{\prime}, \mu^{\prime}, Q^{\prime}, \gamma^{\prime}\right)$ in the category of such cones consists of triples $(f, g, h)$ where $f:(A, \nu) \rightarrow\left(A^{\prime}, \nu^{\prime}\right), g:(B, \mu) \rightarrow\left(B^{\prime}, \mu^{\prime}\right)$, and $h$ is a (again necessarily unique) morphism of $H$-cones above $(f, g)$. We denote the terminal object of this category $\forall_{1}(F, G, H)$, if it exists. By abuse of notation, we denote by $\forall_{1}(F, G, H)$ also the vertex of the $H$-cone in $\forall_{1}(F, G, H)$; it will always be clear from context to which one we refer.

In the rest of the section we show how to interpret $\forall$-types using our axiomatic definition. We show that they support:

(i) A Fibred Semantics: Our axiomatic definitions do not by definition guarantee that the relational interpretation of $\forall X . T$ is a relation between object level interpretations of the same type, so we prove this axiomatically.

(ii) The Identity Extension Lemma: Since we do not "bake-in" the Identity Extension Lemma using for example reflexive graphs, we need to prove it.

(iii) The Abstraction Theorem: We prove the Abstraction Theorem in the axiomatic setting. Most of the work in doing so involves the construction of a model of System $\mathrm{F}$ in the form of a $\lambda 2$-fibration.

We prove the Identity Extension Lemma (iii) for subobject fibrations first, and then generalise it to the case of fibrations which admit full comprehension. The proofs of the fibred semantics (i) and of the Abstraction Theorem (iii) do not require an instantiation to any particular fibration. These proofs are general enough that they can be derived uniformly from Definitions 20 and 21.

\subsection{A Fibred Semantics}

Our proof that if $\vec{R}: \operatorname{Rel}^{n}(\vec{A}, \vec{B})$, then $\llbracket \forall X . T \rrbracket_{1} \vec{R}$ is a relation between $\llbracket \forall X . T \rrbracket_{0} \vec{A}$ and $\llbracket \forall X . T \rrbracket_{0} \vec{B}$ crucially requires opfibrational structure, which plays a distinguishing role in our framework Ghani et al. 2015a].

Lemma 22. Let $F=\left(F_{0}, F_{1}\right)$ and $G=\left(G_{0}, G_{1}\right)$ be pairs of functors $|\mathcal{B}| \rightarrow \mathcal{B}$ and $|\operatorname{Rel}(\mathcal{E})| \rightarrow \operatorname{Rel}(\mathcal{E})$ with $F_{1}$ over $\left(F_{0}, F_{0}\right), G_{1}$ over $\left(G_{0}, G_{0}\right)$, and let $H:|\operatorname{Rel}(\mathcal{E})| \rightarrow \operatorname{Rel}(\mathcal{E})$ with $H$ over $\left(F_{0}, G_{0}\right)$. Then $\forall_{1}(F, G, H)$ is over $\forall_{0} F \times \forall_{0} G$.

Proof. The forgetful functor which maps a fibred $(F, G, H)$-eqcone to its pair of underlying $F$-eqcones and $G$-eqcones is an opfibration, since it inherits the opfibrational structure of $\operatorname{Rel}(U): \operatorname{Rel}(\mathcal{E}) \rightarrow \mathcal{B} \times \mathcal{B}$. For any opfibration $V: \mathcal{D} \rightarrow \mathcal{C}$ which has terminal objects $1_{\mathcal{C}}$ in the base and $1_{\mathcal{D}}$ in the total category, $\Sigma_{!} 1_{\mathcal{D}}$ is isomorphic to $1_{\mathcal{D}}$ and hence we have a terminal object $\Sigma_{!} 1_{\mathcal{D}}$ in $\mathcal{D}$ over $1_{\mathcal{C}}$, where $!: V\left(1_{\mathcal{D}}\right) \rightarrow 1_{\mathcal{C}}$ is the unique morphism from $V\left(1_{\mathcal{D}}\right)$ to the terminal object. Since terminal objects are defined up to isomorphism, we can take $\forall_{1}(F, G, H)$ to be over $\forall_{0} F \times \forall_{0} G$.

This lemma, when taken with the usual treatment of function spaces (and assuming $\forall_{0}$ and $\forall_{1}$ exist), ensures that we have replicated Reynolds' original fibred semantics within our axiomatic framework. That is, for all judgements $\Gamma \vdash T$ Type, $\left(\llbracket T \rrbracket_{0} \times \llbracket T \rrbracket_{0}, \llbracket T \rrbracket_{1}\right)$ forms a fibred functor $|\operatorname{Rel}(U)|^{n} \rightarrow \operatorname{Rel}(U)$. 


\subsection{The Identity Extension Lemma}

The Identity Extension Lemma is, with the Abstraction Theorem, a key property which characterises parametricity. We saw in Section 2 that our axiomatisation gives the right interpretation of $\forall$-types for relations over sets, and the Identity Extension Lemma can be proven from that. The relations over sets arise from a particular fibration of relations obtained via change of base from the subobject fibration over Set. We now show that the Identity Extension Lemma can be proven instantiating our axiomatisation with any subobject bifibration in Section 4.3.1. We then generalise this further to bifibrations with full comprehension in Section 4.3 .2

4.3.1. Subobject Fibrations. In a subobject fibration, Eq : $\mathcal{B} \rightarrow \operatorname{Rel}(\mathcal{E})$ maps an object $X$ to the mono $\left\langle\operatorname{id}_{X}, \operatorname{id}_{X}\right\rangle: X \hookrightarrow X \times X$. Thus we need to show:

Lemma 23. Let $U$ be a subobject bifibration and $\left(F_{0} \times F_{0}, F_{1}\right):|\operatorname{Rel}(U)| \rightarrow \operatorname{Rel}(U)$ a fibred functor. Assume $F=\left(F_{0}, F_{1}\right)$ is equality preserving, i.e. $F_{1} \circ \mathrm{Eq}=\mathrm{Eq} \circ F_{0}$. Then the subobject $\left\langle v_{1}, v_{2}\right\rangle: \forall_{1}\left(F, F, F_{1}\right) \hookrightarrow \forall_{0} F \times \forall_{0} F$ is $\mathrm{Eq}\left(\forall_{0} F\right)=\langle$ id, id $\rangle: \forall_{0} F \hookrightarrow \forall_{0} F \times \forall_{0} F$.

Proof. The heart of the proof is to show that $v_{1}=v_{2}$. To see this, let $\pi_{X}: \forall_{0} F \rightarrow$ $F_{0} X$ be the projection maps associated with $\forall_{0} F$, and let $\gamma_{R}: \forall_{1}\left(F, F, F_{1}\right) \rightarrow F_{1} R$ be the projection maps associated with $\forall_{1}\left(F, F, F_{1}\right)$. By Lemma 22, for every $X, \gamma_{\mathrm{Eq} X}$ : $\forall_{1}\left(F, F, F_{1}\right) \rightarrow F_{1}(\mathrm{Eq} X)=\mathrm{Eq}\left(F_{0} X\right)=F_{0} X$ is over $\left(\pi_{X}, \pi_{X}\right)$. By the definition of the equality functor in a subobject fibration, we have

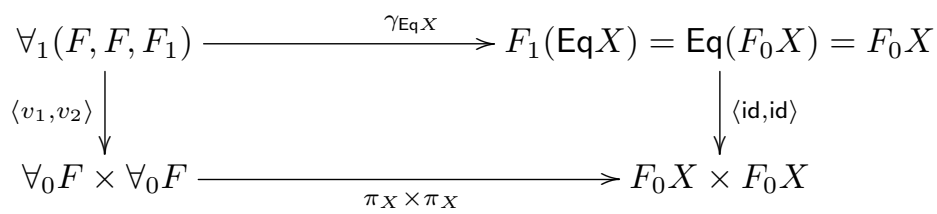

Thus $\pi_{X} v_{1}=\gamma_{\mathrm{Eq} X}=\pi_{X} v_{2}$ and $\left(\forall_{1}\left(F, F, F_{1}\right), \gamma_{\mathrm{Eq}-}\right)$ is a $F$-eqcone with $F_{1}$-cone given by $\gamma_{R}$. Hence both $v_{1}$ and $v_{2}$ are mediating morphisms into the terminal $F$-eqcone, and thus $v_{1}=v_{2}$. Furthermore, they are vertical since $\langle$ id, id $\rangle \circ v_{i}=\left\langle v_{1}, v_{2}\right\rangle$. We can now show that $\mathrm{Eq}\left(\forall_{0} F\right)$ is isomorphic to $\forall_{1}\left(F, F, F_{1}\right)$. In one direction, $\mathrm{Eq}\left(\forall_{0} F\right)$ is easily seen to be a fibred $\left(F, F, F_{1}\right)$-eqcone and hence there is a map of $\operatorname{subobjects} \operatorname{Eq}\left(\forall_{0} F\right) \rightarrow \forall_{1}\left(F, F, F_{1}\right)$. In the other direction, $v_{1}$ is a map of subobjects since $v_{1}=v_{2}$. These maps are mutually inverse, as they are both vertical and the fibration is faithful.

The Identity Extension Lemma for fibred functors $\left(T_{0} \times T_{0}, T_{1}\right):\left.\operatorname{Rel}(U)\right|^{n+1} \rightarrow \operatorname{Rel}(U)$ immediately follows by instantiating $F_{0}=T_{0}(\vec{A},-)$ and $F_{1}=T_{1}(\operatorname{Eq} \vec{A},-)$. When taken with an appropriate treatment of arrow types, this lemma shows that in our axiomatic setting instantiated to subobject fibrations, all type expressions are interpreted not just as fibred functors $\left.\operatorname{Rel}(U)\right|^{n} \rightarrow \operatorname{Rel}(U)$, but as equality preserving fibred functors.

4.3.2. Faithful Bifibrations Which Admit Full Comprehension. We now generalise the previous section to faithful bifibrations which admit full comprehension. In order to do so, we first prove some properties of the comprehension functor. Consider a bifibration 
$\operatorname{Rel}(U): \operatorname{Rel}(\mathcal{E}) \rightarrow \mathcal{B} \times \mathcal{B}$ obtained via change of base as in $\sqrt{3})$. We define the functor $S: \mathcal{E} \rightarrow \operatorname{Rel}(\mathcal{E})$ as follows:

Lemma 24. The map on objects $P \mapsto \Sigma_{\delta_{U} P} P$ extends to a functor $S: \mathcal{E} \rightarrow \operatorname{Rel}(\mathcal{E})$.

Proof. The functor $S$ acts on a morphism $f: P \rightarrow Q$ in $\mathcal{E}$ via the universal property of $\left(\delta_{X}\right)_{\S}$ as in the following diagram

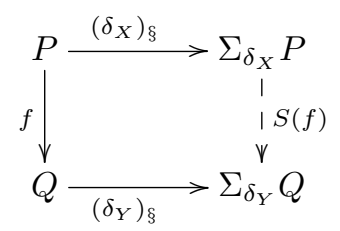

over the diagram

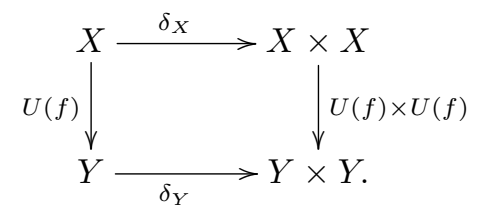

in $\mathcal{B}$ which obviously commutes.

Lemma 25. The functor $S: \mathcal{E} \rightarrow \operatorname{Rel}(\mathcal{E})$ is left adjoint to the projection functor $J: \operatorname{Rel}(\mathcal{E}) \rightarrow \mathcal{E}$ in $(3)$.

Proof. We explicitly give the unit $\eta$ and counit $\epsilon$ of the adjunction since we will use them later. The unit component $\eta_{P}: P \rightarrow \Sigma_{\delta_{U P}} P$ is given by $\eta_{P}=\left(\delta_{U P}\right)_{\S}$. The counit component $\epsilon_{(A, B, R)}:\left(A \times B, A \times B, \Sigma_{\delta_{A \times B}} R\right) \rightarrow(A, B, R)$, is given by $\epsilon_{(A, B, R)}=$ $\left(\pi_{1}, \pi_{2}, p_{A, B}\right)$, where $\pi_{1}$ and $\pi_{2}$ are, respectively, the first and second projections, while $p_{A, B}$ is given by the universal property of $\left(\delta_{A \times B}\right)_{\S}$ with respect to the diagram

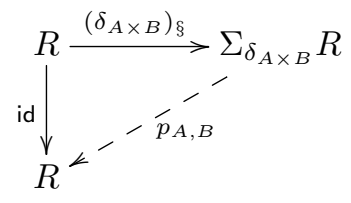

over the commuting diagram

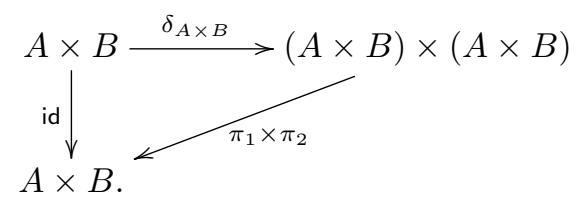

in $\mathcal{B}$. Naturality and the triangle identities follow from direct calculation and bifibrational properties. 
Hence we have a string of adjunctions

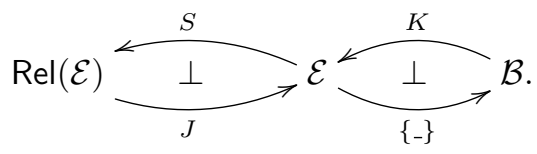

Note that $\mathrm{Eq}=S \circ K$. By composing the adjunctions, we obtain Eq $\dashv\{J(-)\}$. From now on, we will assume that the equality functor is full and faithful. This is a standard assumption in the semantics of parametricity, and corresponds to e.g. the identity condition of Dunphy and Reddy 2004], or very strong equality in Birkedal and Møgelberg 2005]. This assumption is also used elsewhere in order to derive the usual consequences of parametricity in our bifibrational axiomatisation Ghani et al. 2015a.

Before proving the Identity Extension Lemma, we need one more technical result about the comprehension functor.

Lemma 26. If $\mathrm{Eq}$ is full and faithful, then the unit of the adjunction $\mathrm{Eq} \dashv\left\{J\left({ }_{-}\right)\right\}$is an isomorphism $\eta_{A}^{\mathrm{Eq},\{J\}}: A \cong\left\{\Sigma_{\delta} K A\right\}$. Moreover $\pi_{\mathrm{Eq}(A)}=\delta \circ\left(\eta^{\mathrm{Eq},\{J\}}\right)^{-1}$.

Proof. Consider the following diagram:

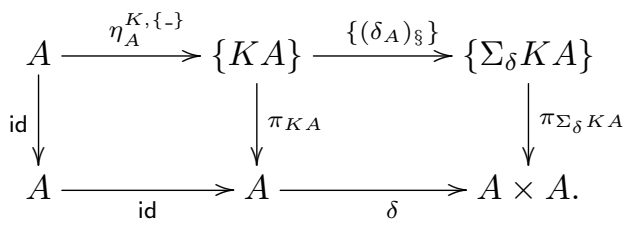

The left square commutes since it arises as the application of $U$ to the triangle identity $\epsilon_{K A}^{K,\{-\}} \circ K \eta_{A}^{K,\{-\}}=$ id. The right square commutes since it is given by $\mathcal{P}\left(\left(\delta_{A}\right)_{\S}\right)$. The morphism $\left(\delta_{A}\right)_{\S}$ is the unit of the adjunction $S \dashv J$, and the composition $\left\{\left(\delta_{A}\right)_{\S}\right\} \circ \eta_{A}^{K,\{-\}}$ is the unit $\eta_{A}^{\mathrm{Eq},\{J\}}$ of the adjunction $\mathrm{Eq} \dashv\{J(-)\}$, which is an isomorphism since Eq is full and faithful by assumption. Hence $\pi_{\mathrm{Eq}(A)}=\delta \circ\left(\eta_{A}^{\mathrm{Eq},\{J\}}\right)^{-1}$.

Lemma 27. Assume Eq is full and faithful. Let $U$ be a bifibration which admits comprehension, and $F=\left(F_{0} \times F_{0}, F_{1}\right):|\operatorname{Rel}(U)| \rightarrow \operatorname{Rel}(U)$ be a fibred functor with $F=\left(F_{0}, F_{1}\right)$ equality preserving, i.e. $F_{1} \circ \mathrm{Eq}=\mathrm{Eq} \circ F_{0}$. Then, if $\forall_{1}\left(F, F, F_{1}\right)$ and $\forall_{0} F$ exist, the morphism $\pi_{\forall_{1} F_{1}}=\left\langle v_{1}, v_{2}\right\rangle:\left\{\forall_{1}\left(F, F, F_{1}\right)\right\} \rightarrow \forall_{0} F \times \forall_{0} F$ is such that $v_{1}=v_{2}$.

Proof. The terminal fibred eqcone comes, for every object $A$ in $\mathcal{B}$, with a morphism $\gamma_{\mathrm{Eq}(A)}$ over $\left(\nu_{A}, \nu_{A}\right)$, which, by Lemma 26 and equality preservation, is sent by $\mathcal{P}$ to the commuting diagram

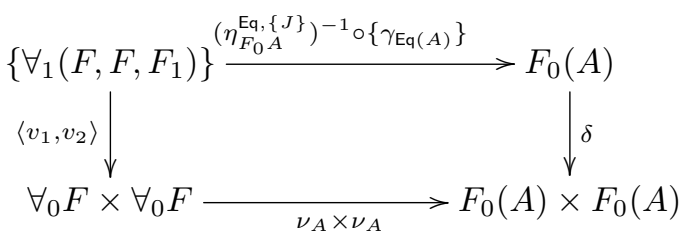

from which we conclude $\nu \circ v_{1}=\nu \circ v_{2}$. 
The system $\left(\left\{\forall_{1}\left(F, F, F_{1}\right)\right\}, \nu_{-} \circ v_{1}\right)$ defines an $F$-eqcone, where the equality cone part is given by precomposing the terminal $F$-eqcone $\left(\forall_{0} F_{0}, \nu\right)$ with $\operatorname{Eq}\left(v_{1}\right): \operatorname{Eq}\left(\left\{\forall_{1} F_{1}\right\}\right) \rightarrow$ $\mathrm{Eq}\left(\forall_{0} F\right)$. It follows that both $v_{1}$ and $v_{2}$ define an eqcone morphism from $\left(\left\{\forall_{1} F_{1}\right\}, \nu \circ v_{1}\right)$ to the terminal eqcone $\left(\forall_{0} F, \nu\right)$, hence $v_{1}=v_{2}$ by uniqueness.

Lemma 28. Assume Eq is full and faithful. Let $U$ be a faithful bifibration which admits full comprehension, and $F=\left(F_{0} \times F_{0}, F_{1}\right):|\operatorname{Rel}(U)| \rightarrow \operatorname{Rel}(U)$ be a fibred functor with $F=\left(F_{0}, F_{1}\right)$ equality preserving, i.e. $F_{1} \circ \mathrm{Eq}=\mathrm{Eq} \circ F_{0}$. The vertex of the $F_{1}$-cone in $\forall_{1}\left(F, F, F_{1}\right)$ is isomorphic to $\operatorname{Eq}\left(\forall_{0} F\right)$, if it exists.

Proof. We give two vertical morphisms $h: \mathrm{Eq}\left(\forall_{0} F\right) \rightarrow \forall_{1}\left(F, F, F_{1}\right)$ and $s: \forall_{1}\left(F, F, F_{1}\right) \rightarrow$ $\mathrm{Eq}\left(\forall_{0} F\right)$ and since the fibration is faithful, their compositions are necessarily identity morphisms.

The terminal $F$-eqcone $\left(\forall_{0} F, \nu\right)$ defines a fibred $\left(F, F, F_{1}\right)$-eqcone whose $F_{1}$-cone vertex is $\operatorname{Eq}\left(\forall_{0} F\right)$ and the vertices of the $F$-eqcones are $\forall_{0} F$. There is an unique morphism $h$ from this cone to $\forall_{1}\left(F, F, F_{1}\right)$ and it is vertical since they are both over $\left(\forall_{0} F, \forall_{0} F\right)$.

For the morphism $s$ in the other direction, by Lemmas 26 and 27, we have a commuting diagram

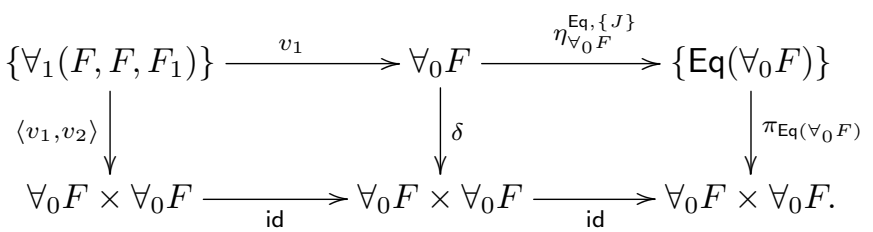

and using fullness and faithfulness of $\mathcal{P}$, there is an unique morphism $s: \forall_{1} F_{1} \rightarrow \operatorname{Eq}\left(\forall_{0} F\right)$ such that $\{s\}=\eta_{\forall 0}^{\mathrm{Eq},\{J\}} \circ v_{1}$ and $U(s)=$ id.

Again, the Identity Extension Lemma for fibred functors $\left(T_{0} \times T_{0}, T_{1}\right):|\operatorname{Rel}(U)|^{n+1} \rightarrow$ $\operatorname{Rel}(U)$ immediately follows by instantiating Lemma 28 with $F_{0}=T_{0}(\vec{A},-)$ and $F_{1}=$ $T_{1}(\mathrm{Eq} \vec{A},-)$. When taken with an appropriate treatment of arrow types, these lemmas show that once again in our axiomatic setting, all type expressions are interpreted not just as fibred functors $|\operatorname{Rel}(U)|^{n} \rightarrow \operatorname{Rel}(U)$, but as equality preserving fibred functors. We now turn to the construction of a model exploiting this fact, from which we derive our axiomatic proof of the Abstraction Theorem.

\subsection{The Abstraction Theorem}

As described in the concrete model in Section 2 , and in the axiomatic framework of Section 4. Reynolds interprets System F types as equality preserving fibred functors. In order to interpret terms - and then establish the Abstraction Theorem - we must therefore discuss models. For our purposes, the notion of a $\lambda 2$-fibration [Seely, 1987 , Jacobs, 1999] as a generic model of System $\mathrm{F}$ is most directly applicable.

Recall that a (split) fibration $p: \mathcal{E} \rightarrow \mathcal{B}$ has a generic object $\Omega \in \mathcal{B}$ if there is a collection of isomorphisms $\theta_{I}: \mathcal{B}(I, \Omega) \cong \mathcal{E}_{I}$, natural in $I$, and that $p$ has simple $\Omega$-products if each reindexing $\pi^{*}$ along a projection $\pi: A \times \Omega \rightarrow A$ has a right adjoint $\Pi_{A}: \mathcal{E}_{A \times \Omega} \rightarrow \mathcal{E}_{A}$ 
such that the Beck-Chevalley condition holds, i.e. $f^{*} \circ \Pi_{B}=\Pi_{A} \circ(f \times \text { id })^{*}$ for every $f: A \rightarrow B$.

Definition 29 ( $\lambda$ 2-fibration). A fibration $p: \mathcal{E} \rightarrow \mathcal{B}$ is a $\lambda 2$-fibration if it is fibred Cartesian closed, $\mathcal{B}$ has finite products, $p$ has a generic object $\Omega \in \mathcal{B}$, and $p$ has simple $\Omega$-products.

We have already taken the first steps towards a model, by showing that types in $n$ free variables can be modelled as equality preserving fibred functors $|\operatorname{Rel}(U)|^{n} \rightarrow \operatorname{Rel}(U)$. We now complete the construction, based upon our axiomatic definitions. We first define the fibres of the $\lambda 2$-fibration, and then the base category:

Construction 30. For each natural number $n$, let the category $\mathcal{F}_{n}^{\mathrm{Eq}}$ have as objects equality preserving fibred functors of the form $\left(F_{0} \times F_{0}, F_{1}\right):|\operatorname{Rel}(U)|^{n} \rightarrow \operatorname{Rel}(U)$. Morphisms are fibred natural transformation of the form $\left(\tau_{0} \times \tau_{0}, \tau_{1}\right):\left(F_{0} \times F_{0}, F_{1}\right) \rightarrow$ $\left(G_{0} \times G_{0}, G_{1}\right)$. Let $\mathcal{L}$ be the category with the natural numbers as objects, and where morphisms $n \rightarrow m$ are $m$-tuples of objects of $\mathcal{F}_{n}^{\mathrm{Eq}}$.

This clearly defines a split fibration $\mathcal{F}^{\mathrm{Eq}} \rightarrow \mathcal{L}$ with reindexing given by composition. The base category has finite products given by addition. In particular, the projection $\pi: n+1 \rightarrow n$ has as $i$ th component the fibred functor $|\operatorname{Rel}(U)|^{n+1} \rightarrow \operatorname{Rel}(U)$ which selects the $i$ th input. By construction, 1 is a generic object. Our previous paper showed how the fibred cartesian closed structure arises from standard structure, e.g. that $\mathcal{B}$ is cartesian closed, $U: \mathcal{E} \rightarrow \mathcal{B}$ is fibred cartesian closed and has simple products, and that the functor Eq has a left adjoint satisfying Frobenius Ghani et al., 2015a, Lemma 4.4]. In the case of subobject fibrations, it is enough to ask that the base $\mathcal{B}$ is a regular LCCC and has coequalisers JJacobs, 1999, Cor. 1.9.9, Prop. 4.8.6, and Prop. 9.2.4.]. All that is left to prove is that we have simple 1-products.

Lemma 31. If terminal fibred eqcones exist, then for each projection $\pi: n+1 \rightarrow$ $n$, the functor $\pi^{*}: \mathcal{F}_{n}^{\mathrm{Eq}} \rightarrow \mathcal{F}_{n+1}^{\mathrm{Eq}}$ has a right adjoint $\Pi=\left(\Pi_{0}, \Pi_{1}\right)$ with $\left(\Pi_{0} F\right) \vec{A}=$ $\forall_{0}\left(F_{0}(\vec{A},-), F_{1}(\mathrm{Eq} \vec{A},-)\right)$ and

$$
\left(\Pi_{1} F\right) \vec{R}=\forall_{1}\left(\left(F_{0}(\vec{A},-), F_{1}(\operatorname{Eq} \vec{A},-)\right),\left(F_{0}(\vec{B},-), F_{1}(\operatorname{Eq} \vec{B},-)\right), F_{1}(\vec{R},-)\right)
$$

and the Beck-Chevalley condition holds.

Proof. We show there is a natural isomorphism between $\pi^{*} G \rightarrow F$ and $G \rightarrow \Pi F$, where $F$ is in $\mathcal{F}_{n+1}^{\mathrm{Eq}}$ and $G$ is in in $\mathcal{F}_{n}^{\mathrm{Eq}}$. Consider a fibred natural transformation $(\tau, \xi)$ : $\pi^{*} G \rightarrow F$. Note that $\left(\pi^{*} G\right)_{0}(\vec{A}, X)=G_{0}(\vec{A})$ for every $X$. Hence $\tau_{\vec{A},-}$ and $\xi_{\mathrm{Eq} \vec{A},-}$ define an $\left(F_{0}(\vec{A},-), F_{1}(\mathrm{Eq} \vec{A},-)\right)$-eqcone with vertex $G_{0}(\vec{A})$, so that there is a map $\rho_{0}: G_{0}(\vec{A}) \rightarrow\left(\Pi_{0} F\right) \vec{A}$ into the terminal such. Similarly $\xi_{\vec{R},-}$ over $\left(\tau_{\vec{A},-}, \tau_{\vec{B},-}\right)$ defines an fibred $\left(\left(F_{0}(\vec{A},-), F_{1}\left(\mathrm{Eq} \vec{A},{ }_{-}\right)\right),\left(F_{0}\left(\vec{B},{ }_{-}\right), F_{1}(\mathrm{Eq} \vec{B},-), F_{1}\left(\vec{R},{ }_{-}\right)\right)\right.$-eqcone and there is an unique morphism $\rho_{1}: G_{1}(\vec{R}) \rightarrow\left(\Pi_{1} F\right) \vec{R}$ which together with $\rho_{0}$ makes up a fibred natural transformation $G \rightarrow \Pi F$. In the other direction, composition with the projections $\left(\nu_{A}, \nu_{B}, \gamma_{R}\right)$ turns natural transformations $G \rightarrow \Pi F$ into natural transformations $\pi^{*} G \rightarrow F$. By the universal property of terminal fibred eqcones, these correspondences 
are mutually inverse. The Beck-Chevalley condition boils down to the fact that both $\left(f^{*} \circ \Pi_{m}\right) F \vec{A}$ and $\left(\Pi_{n} \circ(f \times \mathrm{id})^{*}\right) F \vec{A}$ are defined to be the terminal eqcone for the same functor $F(f \vec{A},-)$.

To summarise, in this section we have proven (using the assumptions from Ghani et al. 2015a, Lemma 4.4]):

Theorem 32. Let $\mathcal{B}$ be cartesian closed, and $U: \mathcal{E} \rightarrow \mathcal{B}$ be a faithful bifibration which is fibred cartesian closed, has simple products, and admits full comprehension. Assume that Eq is full, and has a left adjoint satisfying Frobenius, and assume that terminal fibred eqcones exist. Construction 30 then gives rise to a $\lambda 2$-fibration, where types are interpreted as fibred functors, and terms as fibred natural transformations. By construction, the Abstraction Theorem holds in the sense of (5).

From this theorem, together with Lemma 28, all the usual expected consequences of parametricity - e.g. the existence of initial algebras and final coalgebras, dinaturality follow. See our other paper Ghani et al. 2015a for details and examples of models.

\section{Conclusion}

We have taken Reynolds' definition of the set of parametric polymorphic functions in his relational model, and given an abstract characterisation of it as a universal property in an axiomatic bifibrational framework. Further, we have shown the value of the axiomatisation by proving the two key theorems of parametricity from it, i.e. the Identity Extension Lemma and the Abstraction Theorem. We did this in a fully axiomatic setting of faithful bifibrations which admit full comprehension.

Throughout this paper, we worked with faithful fibrations. Faithfulness is a reasonable assumption, as it corresponds to proof-irrelevant relations - a standing assumption in the literature. We are in the process of lifting this restriction, and thereby tackling proof-relevant parametricity Ghani et al., 2016a, Orsanigo, 2017]. This is a significant undertaking as it involves blending parametricity with higher dimensional cubical structure that, intriguingly, also arises in the semantics of Homotopy Type Theory Bezem et al. 2014 .

\section{References}

Amal Ahmed and Matthias Blume. Typed closure conversion preserves observational equivalence. In International Conference on Functional Programming, ICFP '08, pages 157-168. ACM, 2008.

Amal Ahmed, Derek Dreyer, and Andreas Rossberg. State-dependent representation independence. In Principles of Programming Languages, POPL '09, pages 340-353. ACM, 2009.

Robert Atkey. A deep embedding of parametric polymorphism in Coq. In In Workshop on Mechanising Metatheory, 2009. 
Marc Bezem, Thierry Coquand, and Simon Huber. A model of type theory in cubical sets. In Types for Proofs and Programs (TYPES 2013), volume 26 of Leibniz International Proceedings in Informatics, pages 107-128. Schloss Dagstuhl-Leibniz-Zentrum für Informatik, 2014.

Lars Birkedal and Rasmus E. Møgelberg. Categorical models for Abadi and Plotkin's logic for parametricity. Mathematical Structure in Computer Science, 15:709 - 772, 2005.

Thierry Coquand and Gérard Huet. The Calculus of Constructions. Information and Computation, 76:95 - 120, 1988.

Derek Dreyer, Georg Neis, and Lars Birkedal. The impact of higher-order state and control effects on local relational reasoning. Journal of Functional Programming, 22 (4\&5):477 - 28, 2012.

Brian Dunphy and Uday S. Reddy. Parametric limits. In Logic in Computer Science, LICS '04, pages 242-251. IEEE Computer Society, 2004.

Thomas Ehrhard. A categorical semantics of constructions. In Logic in Computer Science, pages 264-273, 1988.

Neil Ghani, Patricia Johann, Fredrik Nordvall Forsberg, Federico Orsanigo, and Tim Revell. Bifibrational functorial semantics of parametric polymorphism. In Dan R. Ghica, editor, MFPS, 2015a.

Neil Ghani, Fredrik Nordvall Forsberg, and Federico Orsanigo. Parametric polymorphism - universally. In Valeria de Paiva, Ruy de Queiroz, Lawrence S. Moss, Daniel Leivant, and Anjolina G. de Oliveira, editors, Logic, Language, Information, and Computation, volume 9160 of $L N C S$, pages 81-92. Springer, 2015b.

Neil Ghani, Fredrik Nordvall Forsberg, and Federico Orsanigo. Proof-relevant parametricity. In Sam Lindley, Conor McBride, Philip W. Trinder, and Donald Sannella, editors, $A$ List of Successes That Can Change the World - Essays Dedicated to Philip Wadler on the Occasion of His 60th Birthday, volume 9600 of Lecture Notes in Computer Science, pages 109-131. Springer, 2016a.

Neil Ghani, Fredrik Nordvall Forsberg, and Alex Simpson. Comprehensive parametric polymorphism: categorical models and type theory. In Bart Jacobs and Christof Löding, editors, Foundations of Software Science and Computation Structures, volume 9634 of Lecture Notes in Computer Science, pages 3-19. Springer, 2016b.

Jean-Yves Girard, Paul Taylor, and Yves Lafont. Proofs and Types. Cambridge University Press, 1989.

Claudio Hermida. Fibrations, logical predicates and indeterminates. PhD thesis, University of Edinburgh, 1993.

Claudio Hermida. Fibrational relational polymorphism. Available at http://maggie.cs queensu.ca/chermida/papers/FibRelPoly.pdf, 2006.

Chung-Kil Hur and Derek Dreyer. A Kripke logical relation between ML and assembly. In Principles of Programming Languages, POPL '11, pages 133-146. ACM, 2011.

Bart Jacobs. Comprehension categories and the semantics of type dependency. Theoretical Computer Science, 107(2):169-207, 1993.

Bart Jacobs. Categorical Logic and Type Theory. Number 141 in Studies in Logic and the Foundations of Mathematics. North Holland, Amsterdam, 1999. 
F. William Lawvere. Equality in hyperdoctrines and comprehension schema as an adjoint functor. Applications of Categorical Algebra, 17:1-14, 1970.

QingMing Ma and John C. Reynolds. Types, abstractions, and parametric polymorphism, part 2. In Mathematical Foundations of Programming Semantics, pages 1-40. Springer, 1992.

Federico Orsanigo. Bifibrational Parametricity: from zero to two dimensions. PhD thesis, University of Strathclyde, 2017.

Andrew M. Pitts. Polymorphism is set theoretic, constructively. In Category Theory and Computer Science, pages 12-39. Springer, 1987.

Gordon Plotkin and Martìn Abadi. A logic for parametric polymorphism. In TLCA, pages 361 - 375. Springer, 1993.

John C. Reynolds. Types, abstraction and parametric polymorphism. Information Processing, (33), 1983.

John C. Reynolds. Polymorphism is not set-theoretic. In Semantics of Data Types, volume 173 of Lecture Notes in Computer Science, pages 145-156. Springer, 1984.

Robert A.G. Seely. Categorical semantics for higher order polymorphic lambda calculus. Journal of Symbolic Logic, pages 969 - 989, 1987.

Christopher Strachey. Fundamental concepts in programming languages. Lecture Notes, International Summer School in Computer Programming, Copenhagen, 1967. Published in Higher Order Symbolic Computation, 13(1-2):11-49, Kluwer Academic Publishers, 2000.

Stephen Tse and Steve Zdancewic. Translating dependency into parametricity. In International Conference on Functional Programming, ICFP '04, pages 115-125. ACM, 2004.

Philip Wadler. Theorems for free! In Proceedings of the Fourth International Conference on Functional Programming Languages and Computer Architecture, FPCA '89, pages 347-359, 1989. 